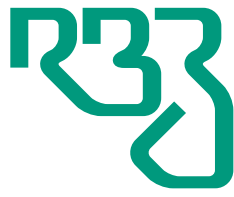

Revista

Brasileira de

Zootecnia

Brazilian Journal of Animal Science

ISSN 1806-9290

www.rbz.org.br

\section{Identification of differentially expressed microRNAs in middle-size ovarian follicles of Meishan and Duroc sows}

\author{
Li-Peng Ma ${ }^{1}$ (iD , Zhi-Chao Zhao ${ }^{2}$, Tao $\mathrm{Li}^{1}$, Da-Quan $\mathrm{Li}^{1}$, Xiao-Yan Wang ${ }^{3}$, \\ Cheng-Yi Song ${ }^{3}$, Ya-Yin $\mathbf{Q i}^{1}$, Tao Huang ${ }^{*}$ (iD \\ ${ }^{1}$ Shihezi University, College of Animal Science and Technology, Shihezi, China. \\ ${ }^{2}$ Guangxi Yangxiang Animal Husbandry Co. Ltd., Guigang, Guangxi, China. \\ ${ }^{3}$ Yangzhou University, College of Animal Science and Technology, Yangzhou, China.
}

\begin{abstract}
This study aimed to investigate the roles of miRNA in the development of ovarian follicles in sows with high ovulation rate. The miRNA expression profiles in middle-size ovarian follicles between Meishan and Duroc sows were characterized by Affymetrix miRNA GeneChip. Seven differentially expressed miRNA were identified. KEGG pathways analyzed the predicted target genes of differentially expressed miRNA by using the DAVID website. The MAPK signaling pathway may affect the process of ovarian follicle development. Our study suggested that miR-21, miR-199a, miR-708, and miR-4334 might play a vital role in the development of ovarian follicles of sow.
\end{abstract}

Keywords: differentially expressed miRNA, follicular phase, middle-sized follicles, sow

\title{
Introduction
}

Ovulation rate is the first factor that limits litter size, and litter size improvement can be efficiently done by selecting sows with high ovulation rate (Petry and Johnson, 2004; Rohrer et al., 1999).

There are differences in the dynamics of follicular growth between prolific and ordinary line during the mid-to-late follicular phase (Driancourt and Terqui, 1996; Miller et al., 1998), and these differences greatly contribute to the high ovulation rate. Gilts of Nebraska Index line selected for high ovulation rate, in turn, potentially changed the dynamics of follicle maturation during the follicular phase of the estrous cycle (Yen, 1999); in addition, a larger pool of medium-sized [medium-sized 1: 3.0-4.9 mm; medium-sized 2: $5.0-6.9 \mathrm{~mm}$ ] follicles were maintained at this stage, and the selection of ovulatory follicles was conducted one day later than the control gilts (Yen et al., 2005). Likewise, the number of follicles in Meishan sows during the follicular phase was higher, while that in the Large-White hybrid sows were decreased between days 16 and 19 (Miller et al., 1998). Besides, the patterns of ovulatory follicular development were different between Large White sows and hyperprolific sows (Driancourt and Terqui, 1996).

The dynamic process that is involved in the follicular phase of estrus cycle would influence the development of follicles and ovulation rate and is controlled by a highly synchronized and exquisitely timed cascade of gene expression (Richards et al., 1995). To better understand the genes that control the ovulation rate and follicular development, differentially expressed genes were identified in the ovarian follicles from a line of pigs selected for indexing ovulation rate, embryo survival, and randomly selected control line (Caetano et al., 2004). Differentially expressed genes in ovarian follicles during the preovulatory stage were identified between Chinese Taihu and Large White sows (Sun et al., 2011). 
However, few studies have revealed the presence of differentially expressed microRNA (miRNA) in medium-sized ovarian follicles.

microRNAare small non-protein-coding transcripts that regulate gene expression post-transcriptionally. Several studies have indicated that miRNA are associated with some ovarian processes by targeting specific genes (Lin et al., 2012; Xu et al., 2011). However, the functions of miRNA in medium-sized ovarian follicles between sows of different breeds remain unknown.

Compared with Duroc pigs, Meishan pigs have larger litter size, which partly result from higher ovulation rate (Bazer et al., 1988; Melville et al., 2002). To develop a deeper insight regarding the gene expression differences in the follicles between prolific sows and western pig breed, the Chinese Meishan and Duroc sows were used to identify the miRNA expression pattern in the M2 ovarian follicles of the follicular phase.

\section{Material and Methods}

All procedures for sample collection were performed according to the protocols approved by the local biological study animal care and use committee in Xinjiang province, China.

Six multiparous Meishan (the third parity) cyclic sows and six multiparous Duroc cyclic sows (the third parity) were selected. The animals were observed twice a day to determine the accurate natural estrous cycle (day 0 is the first day of estrus), and then were injected with PGF2 $\alpha$ analog (cloprostenol, Ningbo Second Hormone Factory) according to the weight of the pigs (the average weights of Meishan and Duroc sows were 100-120 and 250-280 kg, respectively) on day 14 of the estrous cycle to induce luteal regression and synchronize the follicular phase. Ovaries were harvested four days later, and all follicles with diameter $\geq 1 \mathrm{~mm}$ were dissected, snap-frozen in liquid nitrogen (LN2), and stored at $-80{ }^{\circ} \mathrm{C}$ until use. Follicles were classified based on the diameter: small (S: 2-2.9 mm), medium (M1: 3-4.9 mm; M2: 5-6.9 mm), and large ( $\mathrm{L}: \geq 7 \mathrm{~mm}$ ) follicle (Caetano et al., 2004).

Total RNA was extracted from the ovarian follicles of Meishan and Duroc sows by using QIAGEN RNeasy Mini Kit (Qiagen, Hilden, Germany) according to the manufacturer's instructions. The quality and integrity of RNA were determined by $1.2 \%$ agarose gels and detected by the Nanodrop 2000 spectrophotometer (Thermo Scientific, MA, USA).

The Affymetrix GeneChip miRNA 3.0 array analyses (Affymetrix, Santa Clara, CA) were performed by using a commercial Affymetrix array service (Bioassay Laboratory of CapitalBio Corporation, Beijing, China). The Affymetrix GeneChip miRNA3.0 Array contains 19,931 probe sets from 153 organisms including humans, mice, rats, and pigs. The content is derived for sanger miRBase v17 (www.mirbase. org). The RNA labeling, miRNA array hybridization, scanning, and quantification were performed by using Affeymetrix GeneChip system instruments and protocols.

The CEL-files of the raw data were obtained by Affymetrix GeneChip Command Console Software (Affymetrix), and the data was pre-processed by Affymetrix Expression Console software 1.2 (Affymetrix). This included the Robust Multi-array Average (RMA) normalization, subtraction of background intensities, and probe signal integration for the probe Group (the probe set) signal. Significance Analysis of Microarray (SAM) with R package was used to identify differentially expressed miRNA and gene probe sets between M2 ovarian follicles of Meishan and Duroc (Tusher et al., 2001). Probe sets were considered as biologically significant if the fold changes (FC) were $\geq 2$ or $\leq 0.5$ and the q-value was $\leq 5 \%$.

The target genes of the differentially expressed miRNA were the intersection of the predicted genes by using mirwlk2 (validated miRNA-target interactions database) website. The Gene Ontology biological process and KEGG pathway analyses for the predicted targets were performed by using the DAVID (Database for Annotation, Visualization and Integrated Discovery) web-based tool, and all genes in human genome were used as whole genome background for enrichment analysis (Liu et al., 2015).

R. Bras. Zootec., 48:e20170326, 2019 
Cytoscape software was used to predict the interaction between miRNA and predicted target genes (Shannon et al., 2003).

qRT-PCR was used to validate the results of microarray data. Many predicted target genes of the three selected miRNA (ssc-miR-199a, ssc-miR-21 and ssc-miR-708) were involved in the MAPK signaling pathway. Then, ssc-miR-4334 was downregulated in Meishan ovarian follicles. Primers (Table 1) were designed based on miRNA mature sequences. Total RNA of Meishan and Duroc ovarian follicles were extracted by using TRIzol reagent (Invitrogen, CA, USA), and the quality of total RNA was determined by $1.2 \%$ agarose gels and detected by Nanodrop 2000 spectrophotometer (Thermo Scientific, MA, USA). Total RNA were reverse-transcribed into cDNA by using miScriptII RT Kit (QIAGEN, Duesseldorf, Germany) according to the manufacturer's instructions. qRT-PCR was performed by using SYBR Premix Ex TagII (Takara, Dalian, China) in the LightCycler 480 Real-Time PCR machine (Roche, Basel, Switzerland). qRT-PCR conditions were as follows: single cycle of $5 \mathrm{~min}$ at $95^{\circ} \mathrm{C}$, followed by 40 cycles of $30 \mathrm{~s}$ at $95^{\circ} \mathrm{C}, 20 \mathrm{~s}$ at $60^{\circ} \mathrm{C}$, and $15 \mathrm{~s}$ at $72{ }^{\circ} \mathrm{C}$. All experiments were performed in triplicate. The $2^{-\Delta \Delta \mathrm{ct}}$ method was used to determine the differences in expression between Meishan and Duroc sows, and Small nuclear RNA U6 was used as an internal control. The differences in miRNA expression levels between the groups were compared by using two-tailed $t$ test. $\mathrm{P}$ values of $<0.05$ were considered statistically significant.

Table 1 - Primer sequences for qPCR of four randomly selected significantly different miRNA

\begin{tabular}{lcc}
\hline miRNA & Sequence & $\mathrm{Tm}\left({ }^{\circ} \mathrm{C}\right)$ \\
\hline ssc-miR-21 & UAGCUUAUCAGACUGAUGUUGA & 60 \\
ssc-miR-199a & ACAGTAGTCTGCACATTGGTTA & 60 \\
ssc-miR-708 & AAGGAGCTTACAATCTAGCTGGG & 60 \\
ssc-miR-4334 & CCCTGGAGTGACGGGGGTG & 60 \\
\hline
\end{tabular}

The universal reverse primers were provided by miScript II RT Kit (QIAGEN, Duesseldorf, Germany).

\section{Results}

The Affymetrix miRNA microarray was used to detect miRNA expression profiles in M2 ovarian follicle between Meishan and Duroc sows. The 257 probe sets representing the porcine mature miRNA were selected for subsequent analyses. We analyzed the differentially expressed miRNA and identified seven miRNA that showed significant changes in expression (fold change $>2$, adjusted P-value $<0.05$ ) between Meishan and Duroc ovarian follicles library. Six miRNA in Meishan M2 follicle showed significant upregulation (ssc-miR-187, ssc-miR-199a, ssc-miR-19b, ssc-miR-21, ssc-miR-486, and ssc-miR-708), while one was downregulated (ssc-miR-4334) (Table 2).

Four miRNA (ssc-miR-199a, ssc-miR-21, ssc-miR-708, and ssc-miR-4334) were validated by qPCR (Figure 1). The expression patterns were consistent with that of the microarray data. The results showed that ssc-miR-187, ssc-miR-199a, ssc-miR-19b, ssc-miR-21, ssc-miR-486, and ssc-miR-708

Table 2 - Differentially expressed miRNA in M2 ovarian follicles of Meishan and Duroc sows

\begin{tabular}{lcc}
\hline miRNA & LogFC & Adjusted P \\
\hline ssc-miR-187 & 1.72 & 0.0229 \\
ssc-miR-199a & 2.23 & 0.0324 \\
ssc-miR-19b & 1.02 & 0.0417 \\
ssc-miR-21 & 3.20 & 0.0219 \\
ssc-miR-486 & 2.00 & 0.0054 \\
ssc-miR-708 & 1.55 & 0.0305 \\
ssc-miR-4334 & -1.32 & 0.0260
\end{tabular}

LogFC: $\log 2$ (fold-change). 

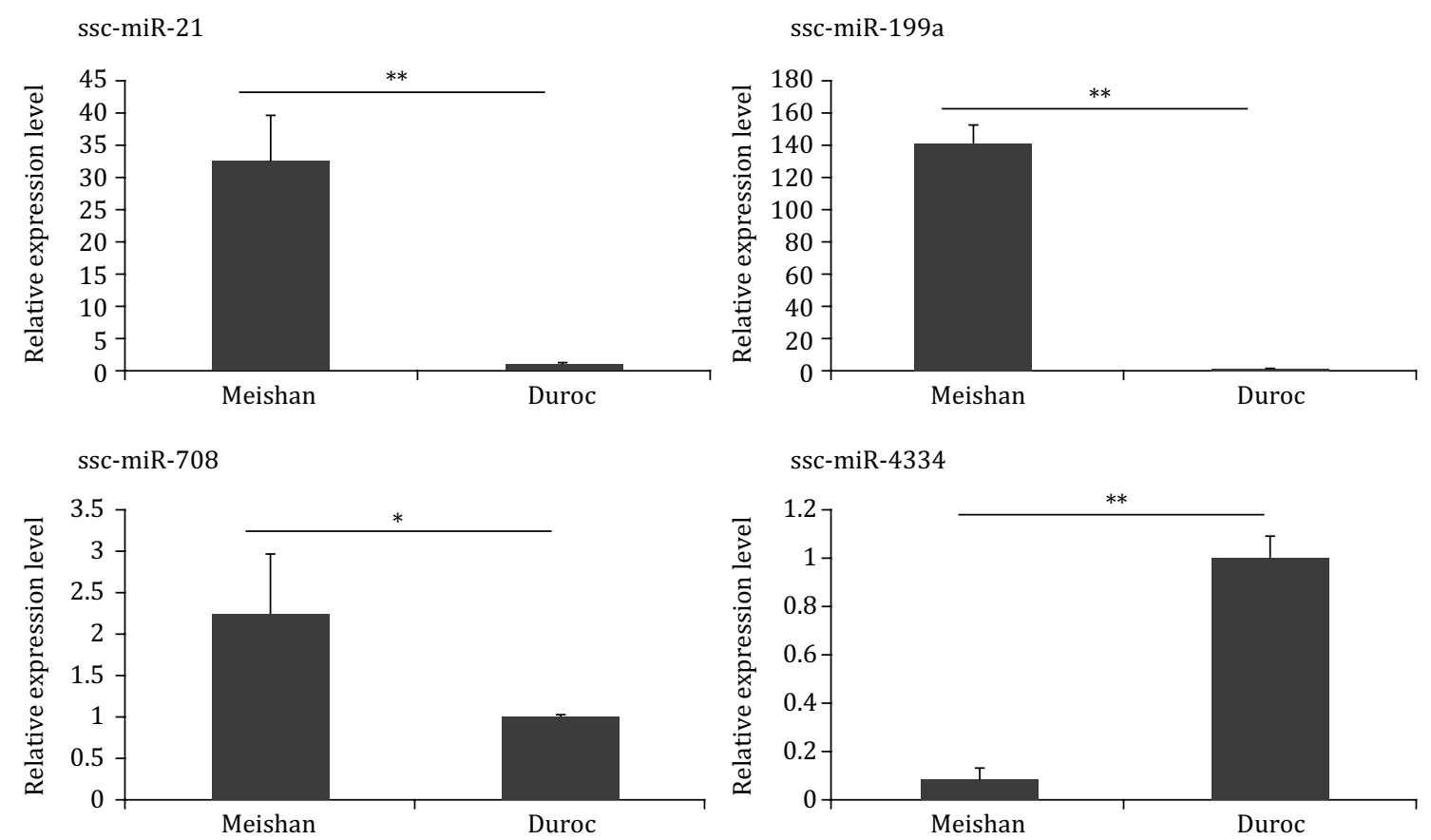

Error bars were presented as SEM (standard error of mean).

The significance of differences for miRNA expression was calculated using the two-tailed t test. $* \mathrm{P}<0.05$.

$* * \mathrm{P}<0.01$.

Figure 1 - Validation of differentially expressed miRNA by qPCR.

were upregulated in Meishan M2 ovarian follicles, while ssc-miR-4334 showed higher expression in the Duroc ovarian follicles.

To understand the function of these differentially expressed miRNA during ovarian follicle development, 1127 target genes of the miRNA were predicted by using mirwlk2 website. Gene ontology analysis based on the predicted targets showed that the differentially expressed miRNA might be involved in response to estrogen stimulus, regulation of cell proliferation, regulation of apoptosis, response to steroid hormone stimulus, and so on (Figure 2).

The KEGG pathway analysis based on the predicted targets revealed differentially expressed miRNA in several signaling pathways, such as mitogen-activated protein kinase (MAPK) signaling pathway, cell cycle, p53 signaling pathway, focal adhesion, progesterone-mediated oocyte maturation, and so on (Figure 3).

\section{Discussion}

This study identified 257 conserved miRNA by microarray analysis in Meishan and Duroc porcine ovarian follicles. Six miRNA were upregulated in Meishan ovarian follicles, while only one, miR-4334, was upregulated in Duroc ovarian follicle.

The Gene Ontology biological process analysis based on the predicted targets showed that these genes were involved in various processes, including response to endogenous stimulus, response to hormone stimulus, regulation of growth, regulation of apoptosis, and regulation of cell proliferation. The development of ovarian follicles might affect the function of steroid hormone secretion from the ovary, such as estrogen, progesterone. It also has direct influence on the normal development of ovaries. In pigs, the process of development of ovarian follicles involves several cell biological processes, such as cell growth, cell motion, and cell apoptosis (Huang et al., 2016). Therefore, our results revealed that miRNA may play an important role in regulating the development of ovarian follicle. 
In our study, MAPK signaling pathway remains the most important pathway and was targeted by three differentially expressed miRNA, including miRNA-21, miRNA-199a, and miRNA-708. The MAPK family proteins, such as p38, ERK, and JNK, play critical roles in porcine granulose cells and oocytes (Shiota et al., 2003; Sriraman et al., 2008; Moreira et al., 2013). Several evidences have suggested that
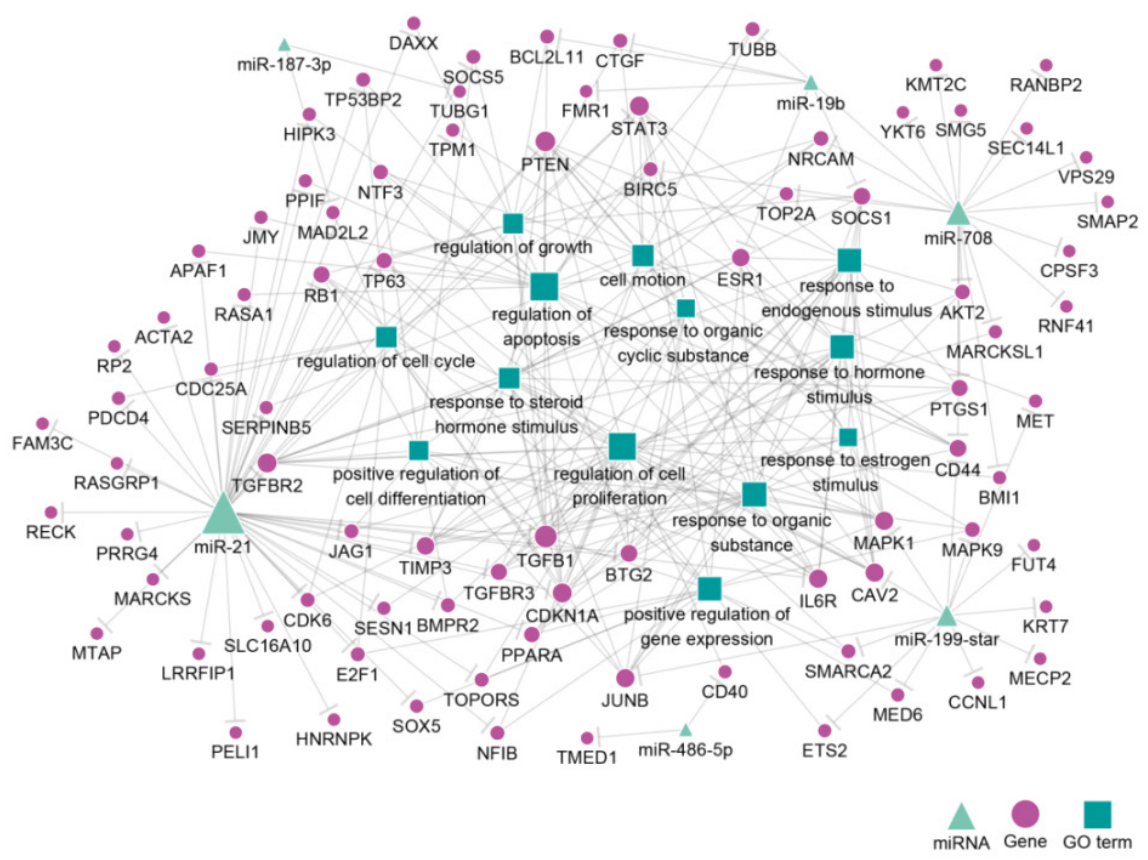

The genes are represented as circles; biological processes are represented as squares; and lines represent the potential relationships between miRNA and genes or the connections between different genes belonging to different biological processes.

The interactions between miRNA and their target genes were predicted by using the bioinformatics software Cytoscape. Green color indicates the upregulated miRNA in Meishan ovarian follicles.

Figure 2 - Interaction network of differentially expressed miRNA and their predicted targets in Meishan and Duroc M2 ovarian follicles.

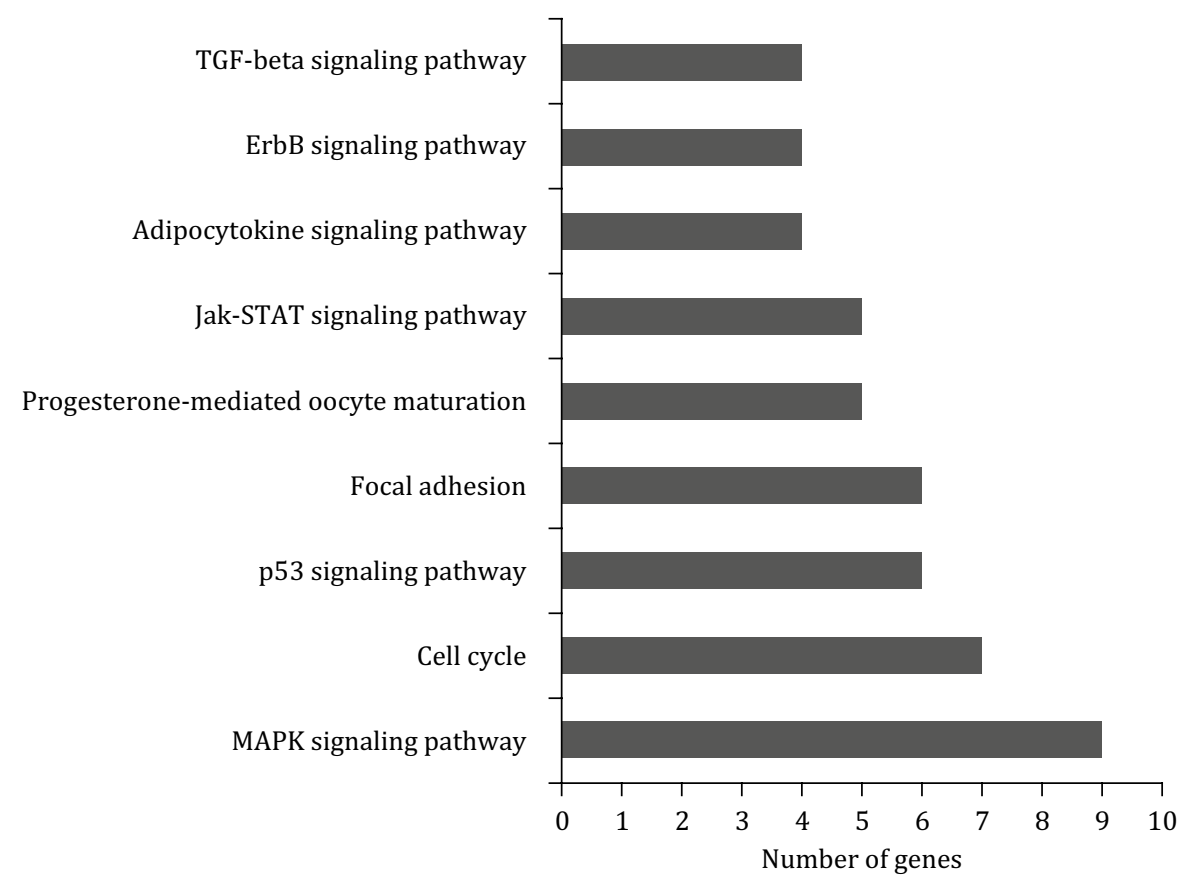

Figure 3 - KEGG pathway enrichment analyses for the predicted targets of the differentially expressed miRNA in Meishan and Duroc M2 ovarian follicles. 
MAPK signaling pathway has an important effect on the regulation of survival or apoptosis of porcine ovarian cells (Shiota et al., 2003; Craig et al., 2004; Li et al., 2008; Moreira et al., 2013). Therefore, further experiments should be performed to elucidate the detailed mechanism that involves MAPK signaling pathway in the regulation of porcine ovarian follicles.

Furthermore, we found that some miRNA targeted well-studied genes that are critical for ovarian follicle development in pigs, such as miR-21/TGFB1, miR-21/TGFBR2, miR-21/TGFBR3, miR-21/BMPR2, miR-19b/ESR1, and miR-199/MAPK1. Transforming growth factor-beta 1 (TGFB1), a member of the TGF family, has demonstrated its presence in granulosa cells of 1-2 mm bovine ovarian follicles, but not in the granulosa cells of 3-5 or 5-10 mm follicles (Nilsson et al., 2003). TGFB1 was first observed in the thecal cells of type 3 (small pre-antral) follicles and also in the thecal of larger follicles, and TGFBR2 was restricted to the thecal cells of type 3 (small pre-antral) and larger follicles (Juengel et al., 2004) in sheep. TGFBR3 showed a high but decreasing mRNA expression level in the populations of human pre-antral follicles (Kristensen et al., 2014). miR-21 also demonstrated to promote the growth of oocytes during ovulation (Yin et al., 2012). These results suggest that the miRNA and their targets of TGF-b family may play an important role in the regulation of ovarian follicle growth and physiology.

ESR1 is the receptor of estrogen. In the bovine ovarian follicles, the mRNA expression of ESR1 was observed in the thecal, stromal, and granulose cells (Schams and Berisha, 2002; Van Den Broeck et al., 2002). Microarray results showed that the expression level of miR-19b was upregulated between Meishan and Duroc pigs. Furthermore, ESR1 was predicted to be a target of miR-19b, implying that it could be a potential regulator of ovarian follicle development in pigs.

Mitogen-Activating Protein Kinase 1 (MAPK1) is localized in the ooplasm of previtellogenic oocytes and plays a functional role in the development and maturation of oocytes/ovaries in P. monodon (Ponza et al., 2011). In mice, the activity of MAPK1 remains essential for the functioning of appropriate granulosa cells during oocyte maturation (Gratao et al., 2008). MAPK1 was predicted to be a target of miR-199. Thus, miR-199 may influence the development and growth of porcine ovarian follicle by targeting MAPK1. Taken together, these findings revealed that the miRNAs regulating the genes have well-known functions in ovarian follicles in pigs.

\section{Conclusions}

The present study revealed the differentially expressed miRNA between M2 ovarian follicles of Meishan and Duroc sows and their predicted targets. The results provided a better understanding regarding the role of miRNA in the development of porcine ovarian follicles.

\section{Conflict of Interest}

The authors declare no conflict of interest.

\section{Author Contributions}

Conceptualization: D.Q. Li and T. Huang. Data curation: L.P. Ma and Z.C. Zhao. Formal analysis: L.P. Ma and Z.C. Zhao. Funding acquisition: T. Huang. Investigation: L.P. Ma, Z.C. Zhao and T. Li. Methodology: L.P. Ma and Z.C. Zhao. Project administration: L.P. Ma and Z.C. Zhao. Resources: X.Y. Wang and C.Y. Song. Software: X.Y. Wang. Supervision: Y.Y. Qi and T. Huang. Validation: L.P. Ma, Z.C. Zhao and T. Li. Writingoriginal draft: L.P. Ma and Z.C. Zhao. Writing-review \& editing: L.P. Ma, Z.C. Zhao and T. Huang.

\section{Acknowledgments}

We acknowledge the National Natural Science Foundation of China (No: 31060295). 


\section{References}

Bazer, F. W.; Thatcher, W. W.; Martinat-Botte, F. and Terqui, M. 1988. Sexual maturation and morphological development of the reproductive tract in Large White and prolific Chinese Meishan pigs. Journal of Reproduction and Fertility 83:723-728. https://doi.org/10.1530/jrf.0.0830723

Caetano, A. R.; Johnson, R. K.; Ford, J. J. and Pomp, D. 2004. Microarray profiling for differential gene expression in ovaries and ovarian follicles of pigs selected for increased ovulation rate. Genetics 168:1529-1537. https://doi.org/10.1534/genetics.104.029595

Craig, J.; Zhu, H.; Dyce, P. W.; Petrik, J. and Li, J. 2004. Leptin enhances oocyte nuclear and cytoplasmic maturation via the mitogen-activated protein kinase pathway. Endocrinology 145:5355-5363. https://doi.org/10.1210/en.2004-0783

Driancourt, M. A. and Terqui, M. 1996. Follicular growth and maturation in hyperprolific and large white sows. Journal of Animal Science 74:2231-2238. https://doi.org/10.2527/1996.7492231x

Gratao, A. A.; Dahlhoff, M.; Sinowatz, F.; Wolf, E. and Schneider, M. R. 2008. Betacellulin overexpression in the mouse ovary leads to MAPK3/MAPK1 hyperactivation and reduces litter size by impairing fertilization. Biology of Reproduction 78:43-52. https://doi.org/10.1095/biolreprod.107.062588

Huang, L.; Yin, Z. J.; Feng, Y. F.; Zhang, X. D.; Wu, T.; Ding, Y. Y.; Ye, P. F.; Fu, K. and Zhang, M. Q. 2016. Identification and differential expression of microRNAs in the ovaries of pigs (Sus scrofa) with high and low litter sizes. Animal Genetics 47:543-551. https://doi.org/10.1111/age.12452

Juengel, J. L.; Bibby, A. H.; Reader, K. L.; Lun, S.; Quirke, L. D.; Haydon, L. J. and McNatty, K. P. 2004. The role of transforming growth factor-beta (TGF-beta) during ovarian follicular development in sheep. Reproductive Biology and Endocrinology 2:78. https://doi.org/10.1186/1477-7827-2-78

Kristensen, S. G.; Andersen, K.; Clement, C. A.; Franks, S.; Hardy, K. and Andersen, C. Y. 2014. Expression of TGF-beta superfamily growth factors, their receptors, the associated SMADs and antagonists in five isolated size-matched populations of pre-antral follicles from normal human ovaries. Molecular Human Reproduction 20:293-308. https://doi.org/10.1093/molehr/gat089

Li, M.; Ai, J. S.; Xu, B. Z.; Xiong, B.; Yin, S.; Lin, S. L.; Hou, Y.; Chen, D. Y.; Schatten, H. and Sun Q. Y. 2008. Testosterone potentially triggers meiotic resumption by activation of intra-oocyte SRC and MAPK in porcine oocytes. Biology of Reproduction 79:897-905. https://doi.org/10.1095/biolreprod.108.069245

Lin, F.; Li, R.; Pan, Z. X.; Zhou, B.; Yu, D. B.; Wang, X. G.; Ma, X. S.; Han, J.; Shen, M. and Liu, H. L. 2012. miR-26b Promotes granulosa cell apoptosis by targeting ATM during follicular atresia in porcine ovary. PLoS ONE 7:e38640. https://doi.org/10.1371/journal.pone.0038640

Liu, R.; Wang, M.; Su, L.; Li, X.; Zhao, S. and Yu, M. 2015. The expression pattern of microRNAs and the associated pathways involved in the development of porcine placental folds that contribute to the expansion of the exchange surface area. Biology of Reproduction 93:62. https://doi.org/10.1095/biolreprod.114.126540

Melville, J. S.; Gibbin, A. M. V.; Robinson, J. A. B.; Gibson, J. P.; Archibald, A. L.; Haley, C. S. and Jiang, Z. 2002. A Meishan positive QTL for prolificacy traits found at the NCOA1 locus on SSC 3. Proceedings of the 7th World Congress on Genetics Applied to Livestock Production, Montpellier, France.

Miller, A. T.; Picton, H. M.; Craigon, J. and Hunter, M. G. 1998. Follicle dynamics and aromatase activity in highovulating Meishan sows and in Large-White hybrid contemporaries. Biology of Reproduction 58:1372-1378. https://doi.org/10.1095/biolreprod58.6.1372

Moreira, F.; Corcini, C. D.; Mondadori, R. G.; Gevehr-Fernandes, C.; Mendes F. F.; Araújo, E. G. and Lucia, T. Jr. 2013. Leptin and mitogen-activated protein kinase (MAPK) in oocytes of sows and gilts. Animal Reproduction Science 139:89-94. https://doi.org/10.1016/j.anireprosci.2013.03.011

Nilsson, E. E.; Doraiswamy, V. and Skinner, M. K. 2003. Transforming growth factor-beta isoform expression during bovine ovarian antral follicle development. Molecular Reproduction and Development 66:237-246. https://doi.org/10.1002/mrd.10350

Petry, D. B. and Johnson, R. K. 2004. Responses to 19 generations of litter size selection in the Nebraska Index line. I. Reproductive responses estimated in pure line and crossbred litters. Journal of Animal Science 82:1000-1006. https://doi.org/10.2527/2004.8241000x

Ponza, P.; Yocawibun, P.; Sittikankaew, K.; Hiransuchalert, R.; Yamano, K. and Klinbunga, S. 2011. Molecular cloning and expression analysis of the Mitogen-activating protein kinase 1 (MAPK1) gene and protein during ovarian development of the giant tiger shrimp Penaeus monodon. Molecular Reproduction and Development 78:347-360. https://doi.org/10.1002/mrd.21310

Richards, J. S.; Fitzpatrick, S. L.; Clemens, J. W.; Morris, J. K.; Alliston, T. and Sirois, J. 1995. Ovarian cell differentiation: a cascade of multiple hormones, cellular signals, and regulated genes. Recent Progress in Hormone Research 50:223-254.

R. Bras. Zootec., 48:e20170326, 2019 
Rohrer, G. A.; Ford, J. J.; Wise, T. H.; Vallet, J. L. and Christenson, R. K. 1999. Identification of quantitative trait loci affecting female reproductive traits in a multigeneration Meishan-White composite swine population. Journal of Animal Science 77:1385-1391. https://doi.org/10.2527/1999.7761385x

Schams, D. and Berisha, B. 2002. Steroids as local regulators of ovarian activity in domestic animals. Domestic Animal Endocrinology 23:53-65. https://doi.org/10.1016/S0739-7240(02)00145-5

Shannon, P.; Markiel, A.; Ozier, O.; Baliga, N. S.; Wang, J. T.; Ramage, D.; Amin, N.; Schwikowski, B. and Ideker, T. 2003. Cytoscape: a software environment for integrated models of biomolecular interaction networks. Genome Research 13:2498-2504. https://doi.org/10.1101/gr.1239303

Shiota, M.; Sugai, N.; Tamura, M.; Yamaguchi, R.; Fukushima, N.; Miyano, T. and Miyazaki, H. 2003. Correlation of mitogen-activated protein kinase activities with cell survival and apoptosis in porcine granulosa cells. Zoological Science 20:193-201. https://doi.org/10.2108/zsj.20.193

Sriraman, V.; Modi, S. R.; Bodenburg, Y.; Denner, L. A. and Urban, R. J. 2008. Identification of ERK and JNK as signaling mediators on protein kinase C activation in cultured granulosa cells. Molecular and Cellular Endocrinology 294:52-60. https://doi.org/10.1016/j.mce.2008.07.011

Sun, X.; Mei, S.; Tao, H.; Wang, G.; Su, L.; Jiang, S.; Deng, C.; Xiong, Y. and Li, F. 2011. Microarray profiling for differential gene expression in PMSG-hCG stimulated preovulatory ovarian follicles of Chinese Taihu and Large White sows. BMC Genomics 12:111. https://doi.org/10.1186/1471-2164-12-111

Tusher, V. G.; Tibshirani, R. and Chu, G. 2001. Significance analysis of microarrays applied to the ionizing radiation response. Proceedings of the National Academy of Sciences of United States of America 98:5116-5121. https://doi.org/10.1073/pnas.091062498

Van Den Broeck, W.; Coryn, M.; Simoens, P. and Lauwers, H. 2002. Cell-specific distribution of oestrogen receptor-alpha in the bovine ovary. Reproduction in Domestic Animals 37:291-293. https://doi.org/10.1046/j.1439-0531.2002.00352.x

$\mathrm{Xu}$, S.; Linher-Melville, K.; Yang, B. B.; Wu, D. and Li, J. 2011. Micro-RNA378 (miR-378) regulates ovarian estradiol production by targeting aromatase. Endocrinology 152:3941-3951. https://doi.org/10.1210/en.2011-1147

Yen, H.-W. 1999. Follicular development, maturation and atresia during the estrous cycle in gilts expressing high or low ovulation rate.

Yen, H.-W.; Ford, J. J.; Zimmerman, D. R. and Johnson, R. K. 2005. Follicular development and maturation in gilts selected for an index of high ovulation rate and high prenatal survival. Journal of Animal Science 83:130-135. https://doi.org/10.2527/2005.831130x

Yin, M.; Lu, M.; Yao, G.; Tian, H.; Lian, J.; Liu, L.; Liang, M.; Wang, Y. and Sun, F. 2012. Transactivation of microRNA-383 by steroidogenic factor-1 promotes estradiol release from mouse ovarian granulosa cells by targeting RBMS1. Molecular Endocrinology 26:1129-1143. https://doi.org/10.1210/me.2011-1341 